\title{
Fetus in fetu, presentación de dos casos: diagnóstico diferencial y revisión de la literatura
}

Fetus in Fetu, a Two-case Presentation: Differential Diagnosis and Literature Review

Fetus in fetu, apresentação de dois casos: diagnóstico diferencial e revisão da literatura

Federico Lubinus, MD., Esp. ${ }^{1}$ (iD, Evelin Zuñiga Hadechni, MD., Esp. ${ }^{2}$ iD, Luis Andrés López Martínez, MD. ${ }^{3}$ iD, César Andrés Rueda Hernández, MD. ${ }^{4}$ iD

1. Médico Radiólogo. Miembro del Staff de Radiólogos Especializados Clínica FOSCAL. Director Académico del programa de Radiología e Imágenes Diagnósticas, Universidad Autónoma de Bucaramanga. Bucaramanga, Santander, Colombia.

2. Médico Radiólogo. Miembro del Staff de Radiólogos Especializados Clínica FOSCAL, Floridablanca, Santander, Colombia.

3. Residente de Radiología e Imágenes Diagnósticas. Universidad Autónoma de Bucaramanga. Bucaramanga, Santander, Colombia.

4. Médico. Servicio Social Obligatorio FOSCAL, Floridablanca, Santander, Colombia.

Correspondencia. Federico Lubinus. Universidad Autónoma de Bucaramanga. Email.

\section{INFORMACIÓN DEL ARTÍCULO:}

Artículo recibido: 30 de agosto de 2019

Artículo aceptado: 16 de julio de 2020

DOI: https://doi.org/10.29375/01237047.3685

Cómo citar: Lubinus F, Zuñiga-Hadechni E, López L, Rueda-Hernández, CA. Fetus in fetu, presentación de dos casos: diagnóstico diferencial y revisión de la literatura. MedUNAB. 2020;23 (3): 464-470. doi: https://doi. org/10.29375/01237047.3685

\section{RESUMEN}

Introducción. Fetus in fetu es una rara entidad congénita en la cual se presenta un gemelo monocigótico que en la mayoría de los casos está incorporado en el abdomen del hermano. La presentación más frecuente es una masa retroperitoneal. La diferenciación diagnóstica entre fetus in fetu y teratoma es discutida. El objetivo de este trabajo es describir el caso de dos pacientes con fetus in fetu, mediante una revisión reducida de la literatura en sus hipótesis etiológicas y métodos 
diagnósticos. Reporte de casos. Se presentan 2 casos, el primer caso es un lactante menor con distensión abdominal persistente, en quien se palpó masa abdominal. El segundo caso es un recién nacido con diagnóstico prenatal de masa abdominal. Ambos casos presentan imágenes radiológicas que evidencian masa abdominal compatible con fetus in fetu, razón por la cual posteriormente fueron intervenidos quirúrgicamente para resección de la masa. Discusión. Es importante tener presente esta patología como diagnóstico diferencial de masa abdominal en los pacientes infantes, ya que el poder diferenciarlo de un teratoma maduro nos indicaría que se trata de una alteración del desarrollo embriológico y no de una patología tumoral. Para esto, son necesarios métodos imagenológicos y en algunos casos pruebas cromosómicas o histopatológicas, con el fin de esclarecer el diagnóstico del fetus in fetu. Conclusión. El fetus in fetu es una patología rara con pocos casos diagnosticados prenatalmente, la cual se diagnostica principalmente en la infancia. Las imágenes radiológicas y en algunos casos las pruebas cromosómicas o histopatológicas, son de gran importancia para el diagnóstico definitivo.

Palabras claves:

Fetus in fetu; Teratoma maduro; Rayos X; Tomografía axial computarizada por Rayos X; Imagen por Resonancia Magnética; Ultrasonografía.

\section{ABSTRACT}

Introduction. Fetus in fetu is a rare congenital condition that has been defined as the presence of one monozygotic twin, in most cases, in the sibling's abdomen. It is most frequently present as a retroperitoneal mass. The diagnostic difference between fetus in fetu and teratoma is discussed. This work's objective is to describe the case of two patients with fetus in fetu through a reduced literature review in terms of etiologic hypotheses and diagnostic methods. Case Reports. Two cases are presented. The first case is that of an infant with persistent abdominal distension, in whom an abdominal mass was found by palpating. The second case is that of a newborn baby with a prenatal abdominal mass diagnosis. Both cases present X-ray images that demonstrate an abdominal mass compatible with fetus in fetu, reason why the patients were subsequently surgically intervened to remove the mass. Discussion. It is important to keep this pathology in mind as a differential diagnosis of abdominal masses in infant patients, since being able to differentiate it from a mature teratoma would indicate that the mass is an embryonic development alteration, not a tumor pathology. Imaging methods and, in some cases, chromosome or histopathological analyses, are necessary for this, in order to clarify the diagnosis of fetus in fetu. Conclusion. Fetus in fetu is a rare pathology with few cases diagnoses prenatally. It is mainly diagnosed during infancy. X-ray images and, in some cases, chromosome or histopathological analyses, are greatly important for definitive diagnoses.

Keywords:

Fetus in fetu; Teratoma; X-rays; Tomography, X-Ray Computed; Magnetic Resonance Imaging; Ultrasonography.

\section{RESUMO}

Introdução. Fetus in fetu é uma entidade congénita rara em que existe um gémeo monozigótico que, na maioria dos casos, está incorporado no abdómen do irmão. A apresentação mais comum é uma massa retroperitoneal. A diferenciação diagnóstica entre fetus in fetu e teratoma é discutida. $\mathrm{O}$ objetivo deste trabalho é descrever o caso de dois pacientes com fetus in fetu, por meio de uma revisão reduzida da literatura sobre as suas hipóteses etiológicas e métodos de diagnóstico. Relato de casos. Dois casos são apresentados, o primeiro, é um bebê menor com distensão abdominal persistente, no qual foi sentida uma massa abdominal. O segundo caso é um recém-nascido com diagnóstico pré-natal de massa abdominal. Ambos os casos apresentam imagens radiológicas mostrando massa abdominal compatível com fetus in fetu, razão pela qual foram posteriormente operados para ressecção da massa.

Discussão. É importante considerar esta patologia como diagnóstico diferencial da massa abdominal em pacientes infantis, uma vez que ser capaz de diferenciá-la de um teratoma maduro indicaria que se trata de uma alteração no desenvolvimento embriológico e não de uma patologia tumoral. Para isso, são necessários métodos de imagem e em alguns casos testes cromossômicos ou histopatológicos, a 
fim de esclarecer o diagnóstico do fetus in fetu. Conclusão. Fetus in fetu é uma patologia rara que é diagnosticado principalmente na infância, com poucos casos diagnosticados no pré-natal. As imagens radiológicas e, em alguns casos, os testes cromossômicos ou histopatológicos são de grande importância para o diagnóstico definitivo.

Palavras-chave:

Fetus in fetu; Teratoma maduro; Raios-X; Tomografia Computadorizada por Raios X; Ressonância magnética; Ultrassonografia.

\section{Introducción}

Fetus in fetu (FIF) es una rara entidad que ocurre aproximadamente en 1 en 500,000 nacidos vivos y se define como un gemelo monocigótico incorporado dentro del abdomen de su hermano durante el desarrollo. El término fue introducido por Johann Friedrich Meckel en 1800, mientras que el primer reporte se citó en 1809 $(1,2)$ y desde entonces se han reportado menos de 200 casos en la literatura. Desde finales del siglo XVIII hasta el siglo XX se reportaron alrededor de 87 casos y en los últimos 17 años se han publicado aproximadamente otros 100 casos adicionales (3).

Durante gran parte del siglo XIX y principios del siglo XX, se creía que el FIF era un teratoma maduro $(4,5)$. Willis, con la intención de distinguir estas dos patologías, sugiere que un FIF está conformado por una masa que contiene un eje vertebral en una masa encapsulada, pedunculada, del tipo fetiforme, con estructuras desorganizadas alrededor de este eje. Está frecuentemente situado en el área retroperitoneal, alrededor de la cual se encuentran órganos $(5,6)$.

La gran mayoría de autores refieren que el FIF es el resultado de una inadecuada embriogénesis en embarazos monocoriales biamnióticos debido a una inadecuada división o implantación del blastocisto y su desenlace es un feto vertebrado que se encuentra encerrado dentro de un feto con desarrollo normal (7). Mientras que otros autores sugieren que es un teratoma que varía de inmaduro a maduro $(6, \mathrm{p} 2)$. El teratoma maduro es un tumor de células germinales definido histológicamente por la presencia de las tres capas germinales: ectodermo, endodermo y mesodermo (la grasa es el principal marcador de su origen mesodérmico), siendo su localización en niños más habitual a nivel sacrocoxígeo. Otra localización puede ser retroperitoneal donde frecuentemente son bien diferenciados (8), localización en la cual es más frecuentemente encontrado el FIF. Por tanto, la distinción entre estas patologías en algunos casos suele ser difícil. Se presentan a continuación dos casos típicos de FIF que se presentaron en el servicio de
Radiología e Imágenes Diagnósticas en un centro de alta complejidad con el objetivo de resaltar la importancia de tener en cuenta el FIF como diagnóstico diferencial en pacientes infantes con presencia de masa abdominal, planteando una correlación clínico-imagenológica en la diferenciación del FIF.

\section{Casos clínicos}

\section{Caso 1}

Niña de 3 meses de edad, quien llega a consulta médica traída por la mamá por distensión abdominal persistente, sin ningún otro síntoma adicional, con tiempo de evolución desconocido. Se encuentra al examen físico masa palpable que ocupa casi la totalidad del abdomen, resto del examen físico sin hallazgos patológicos $\mathrm{Se}$ realizó estudio de ultrasonido, el cual reveló una masa de ecoestructura heterogénea con componente sólido y quístico, con calcificaciones en su interior, sugestivas de FIF vs. teratoma maduro (figura 1). La resonancia magnética (RMN) abdominal mostró masa de intensidad de señal heterogénea de apariencia fetal con extremidades rudimentarias, sin evidencia de restricción en las secuencias de difusión, hallazgos compatibles con FIF (figura 3). Fue llevada a cirugía, en donde se encuentra masa con presencia de cabeza, tronco, extremidades con falanges rudimentarias, esbozo de orejas y cara (imagen 2). Paciente evolucionó de manera satisfactoria. Se obtuvo consentimiento informado de los padres para publicación del caso.

\section{Caso 2}

Recién nacido con diagnóstico prenatal de masa abdominal (FIF vs. teratoma maduro), no se tienen imágenes del diagnóstico prenatal. El feto evolucionó normalmente, con curvas de crecimiento dentro de los límites normales, trabajo de parto sin complicaciones. $\mathrm{Al}$ examen físico neonatal se palpa masa que ocupa casi la totalidad del abdomen, por lo demás del examen físico normal. En la tomografía axial computarizada (TAC) 
Figura 1. Ultrasonido abdominal.
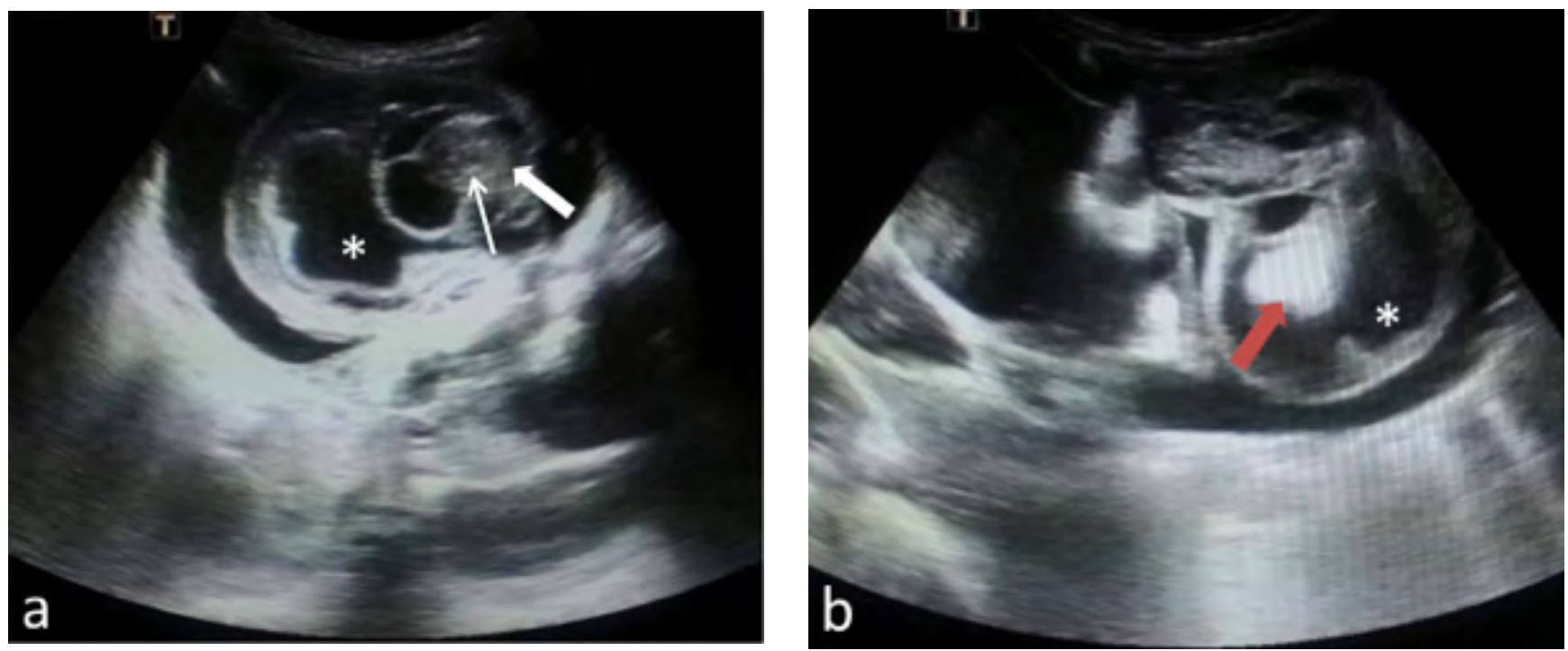

Fuente: departamento de Radiología de la entidad clínica.

Nota: Imagen a: muestra una masa compleja sólida con una cápsula con contenido líquido, correspondientes a su bolsa y líquido amniótico. También se identifica una zona quística señalada por el asterisco. En el componente sólido de la masa se observan calcificaciones señaladas con flecha delgada. Imagen b: se evidencia contenido encefálico señalado con flecha, rodeada de líquido que aparentemente corresponde a saco gestacional con feto, con alteraciones morfológicas e hidranencefalia señalado con asterisco vs. teratoma maduro.

Figura 2. TAC abdominal corte axial

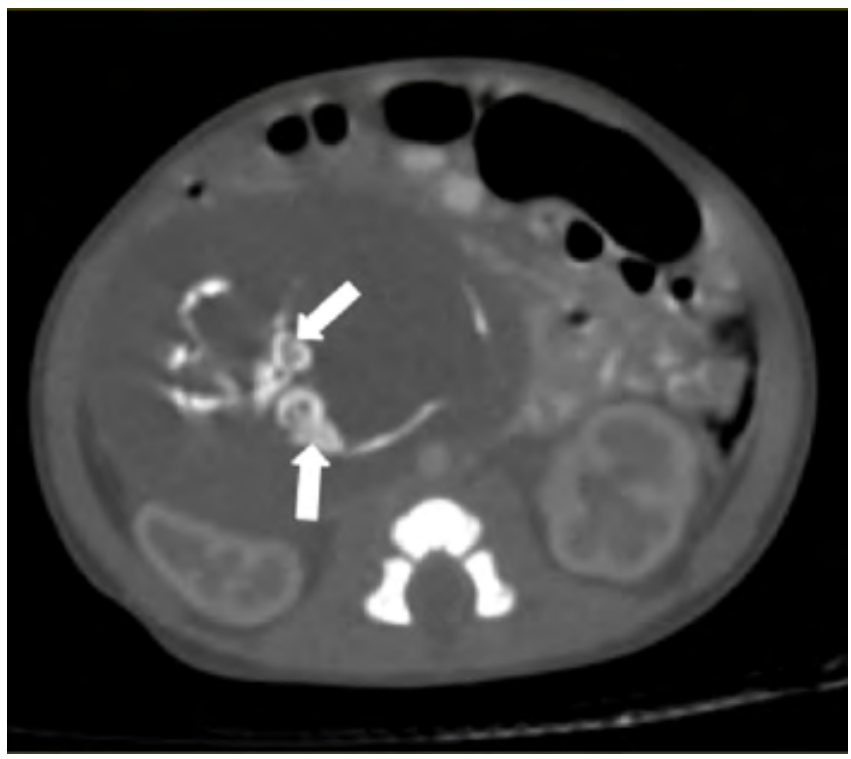

Fuente: departamento de Radiología de la entidad clínica.

Nota: se evidencia masa redondeada, bien definida, en peritoneo, que causa moderados signos de compresión, de los órganos abdominales, como el riñón y el hígado, con presencia de estructuras calcificadas, en relación con componente óseo, señalados por flecha blanca. de abdomen se evidenció una masa de localización peritoneal, bien definida, con presencia de estructuras óseas que asemejan vértebras; compatible con un FIF (figura 2). Fue llevado a cirugía, en donde se encuentra masa con presencia de extremidades inferiores con falanges rudimentarias, esbozos de miembros superiores y genitales externos mal desarrollados (imagen 1). Paciente evolucionó de manera satisfactoria. Se obtuvo consentimiento informado de los padres para publicación del caso.

\section{Discusión}

La etiología del FIF no es clara aún. Hay dos hipótesis que podrían explicar la formación del FIF. La primera hipótesis, que es la más reportada en la literatura, habla acerca de embarazos gemelares monocigóticos, monocoriónicos, diamnióticos, en donde se cree que el gemelo parásito se incluye en el gemelo huésped durante el proceso de plegamiento ventral del disco embrionario trilaminar (9). Se especula que la inclusión en el hermano huésped es por una persistencia de la anastomosis de la circulación vitelina durante el desarrollo $(10,11)$. La arteria mesentérica superior se desarrolla a partir de la circulación vitelina, lo que explica la alta frecuencia de localización del FIF en retroperitoneo superior. 
Figura 3. Imagen a. RMN abdominal en secuencia $\mathrm{T} 2$ corte axial; Imagen $\mathrm{b}$. Secuencia $\mathrm{T} 1$ corte coronal
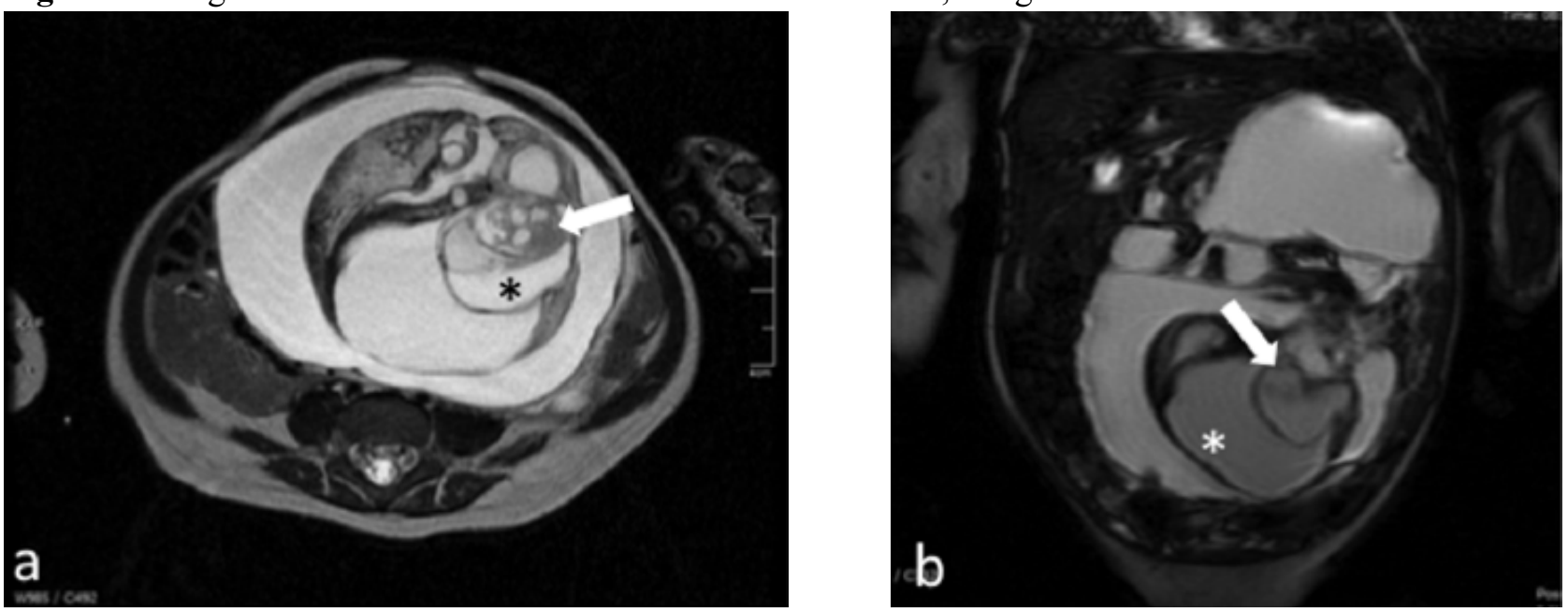

Fuente: departamento de Radiología de la entidad clínica.

Nota: imagen a: se evidencia una masa retroperitoneal compleja bien definida que desplaza el contenido abdominal señalado por flecha, con apariencia fetal, hidranencefalia señalada por asterisco. Imagen b: se evidencia masa bien definida con presencia de extremidades rudimentarias señalado por flecha tronco parcialmente desarrollado señalado por asterisco.

Por otro lado, en el útero de la madre el crecimiento de los gemelos es paralelo, pero debido a la dominancia vascular del gemelo huésped o a un defecto intrínseco del gemelo parásito, termina por generar una parada repentina del crecimiento en el gemelo parásito $(12,13)$, lo que conduce a una masa mal formada con varias estructuras desarrolladas parcialmente, como se reporta en nuestros dos casos presentados y que se localizan con mayor frecuencia en el retroperitoneo.

La mayoría de los FIF son anencefálicos, con presencia de columna vertebral en un $91 \%$ y extremidades en un $82.5 \%$. Con mayor frecuencia, los miembros inferiores son más desarrollados que los miembros superiores $(3, \mathrm{p} 2)$. La segunda hipótesis se propone en embarazos de doble fecundación en el cual un blastocisto alrededor del quinto día postfecundación es implantado dentro del otro blastocisto en lugar de la implantación normal en el endometrio uterino; tal implantación no se desarrolla normalmente, lo que origina un FIF $(7, \mathrm{p} 2)$. Por otra parte, algunos autores refieren que dicha anomalía podría ser un teratoma maduro y no un FIF, razón por la cual el FIF es una patogenia muy controvertida (14).

El $80 \%$ de los casos de FIF son encontrados en retroperitoneo (15), hay otros lugares atípicos de presentación como: intracraneales (16), glándulas adrenales (17), intratorácicos (18), cuello (19), ovario (20), orofaringe (21) y escroto (22).
El diagnóstico puede ser hecho por Rayos-X, TAC, RMN, ultrasonografía, en los cuales se evidencia la presencia de huesos de miembros o vértebras parcialmente desarrollados, esbozos de extremidades rudimentarias y órganos conocidos como los signos de Meckel (23), los cuales pueden ser observados en la patología de manera macroscópica (imagen 1, imagen 2) y que son reportados en ambos casos del presente artículo en las imágenes radiológicas (figura 1, figura 2, figura 3). La visualización de una masa no homogénea con huesos, especialmente vértebras, es considerado el signo más patognomónico del FIF. El diagnóstico es principalmente en infantes, pero también se han reportado casos de diagnóstico antenatal en los que se evidencia como hallazgo principal a la ultrasonografía, la presencia de vértebras acompañada de masa heterogénea. En estos casos el diagnóstico se hace en estadios finales del tercer trimestre (24).

La histopatología podría confirmar el diagnóstico, además el cariotipo, marcadores serológicos y mapeo de restricción de $\mathrm{ADN}(9, \mathrm{p} 3)$ podrían también ayudar a definir la patología en algunos casos tales como FIF pobremente diferenciados. Aunque es una patología benigna, hay un caso reportado de malignidad en el que se produjo a los 4 meses de la extracción completa del FIF una recurrencia manifestada como un tumor del saco vitelino. Después de la resección quirúrgica de dicha masa, fue tratado con quimioterapia y recuperado a los 2 años de edad (25). 
El tratamiento de FIF es una resección completa de la masa mediante cirugía, tal y como se llevó a cabo en los dos casos reportados en este artículo (26). Blumber NA reporta un caso en el cual la resección de la masa fue subtotal, ya que se encontraba adherida a órganos internos, lo que hizo difícil su completa extracción (27). El objetivo principal de la cirugía es la eliminación de los síntomas de masa abdominal causados por el FIF tales como: distensión abdominal, masa palpable, emesis, pobre alimentación y disnea $(20, \mathrm{p} 4)$, síntomas que fueron reportados en ambos pacientes del presente artículo.

De ninguna manera un teratoma podría llegar a tener un grado de diferenciación tal, con morfología tan cercana a un feto como lo observamos en nuestro caso 1 (imagen 2), y desarrollo de estructuras anatómicas como el caso 2 (imagen 1); esto apoyado en el hecho de no reportes de recurrencia en la literatura, indica que se trata de una alteración del desarrollo embriológico y no de una patología tumoral. Por otro lado, si el desarrollo embriológico es muy deficiente, en el contexto de una masa neonatal con derivados de las tres capas germinales, un teratoma podría ser un FIF mal diferenciado, lo que haría pensar que la presencia de una masa con columna vertebral como criterio diagnóstico de FIF sería arbitrario, y se estarían diagnosticando muchos teratomas neonatales que son realmente FIF pobremente diferenciados.

Figura 4. Imagen 1. (Caso 2): pieza quirúrgica compuesta por masa con presencia de extremidades inferiores con formación de dedos, vestigios de miembros superiores y genitales externos mal desarrollados. Imagen 2. (Caso 1): nótese la presencia de cabeza, tronco, extremidades con falanges rudimentarias, esbozo de orejas y cara.
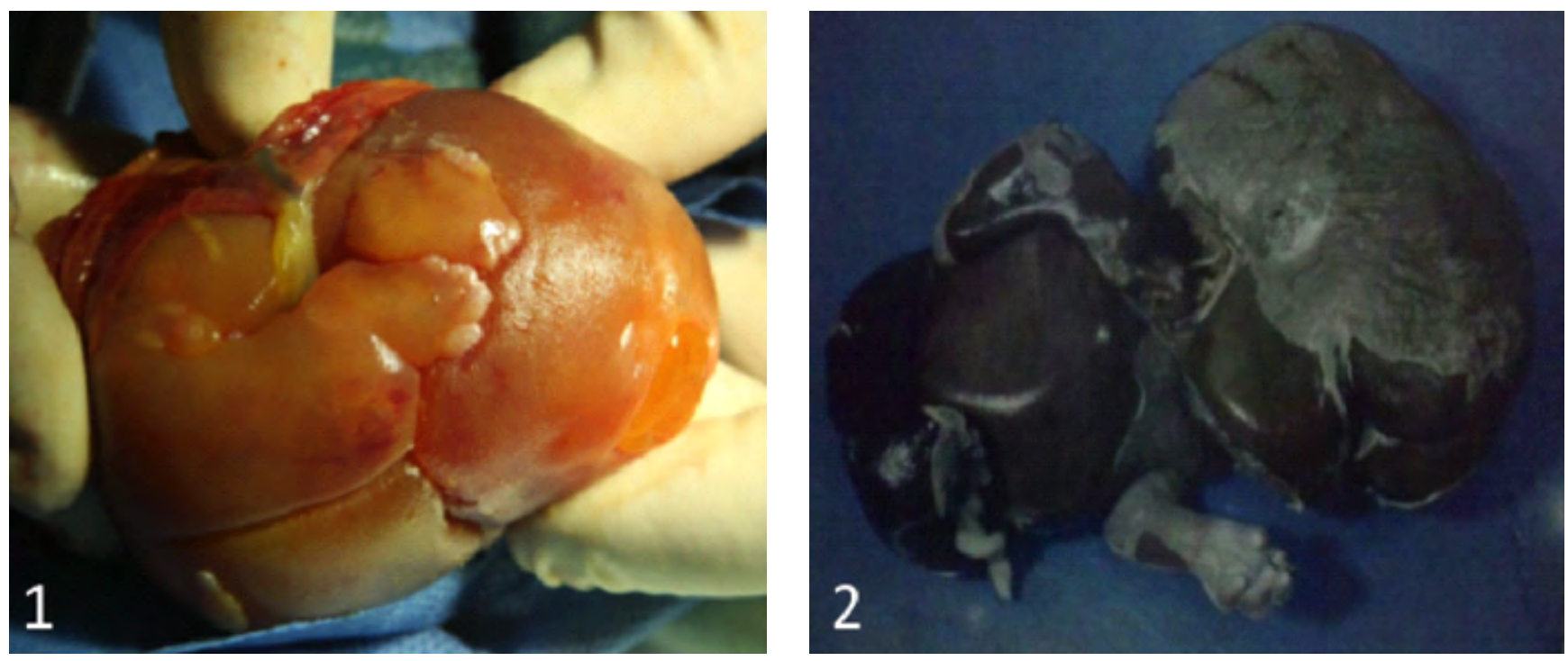

Fuente: patología y Citología Dr. Kleber Zamora y Dr. Reinel Sanjuan.

\section{Conclusión}

El FIF es una patología infrecuente que se presenta en la infancia, principalmente. Se presenta clínicamente como masa abdominal, que supone un reto diagnóstico en el personal de salud. Ante la presentación de un infante con masa abdominal, se debe pensar en FIF, ya que hace parte del espectro de masas abdominales en pacientes pediátricos. Por último, se deben solicitar imágenes radiológicas con el fin de esclarecer el diagnóstico de FIF, pero lo anterior podría limitar el diagnóstico de FIF, ya que no siempre se evidencian signos radiológicos que orienten al diagnóstico de dicha patología, como en el caso de los FIF mal diferenciados. En estos casos los métodos histopatológicos o cromosómicos (cariotipo, marcadores serológicos o mapeo de restricción ADN) podrían ser de gran ayuda para generar el diagnóstico. Sin embargo, con el avance del diagnóstico prenatal se espera que un mayor número de casos se logren diagnosticar de manera prenatal y de esta forma, se puede planear una resección más temprana y así limitar la posibilidad de malignidad que ha sido descrita en la literatura. 


\section{Referencias}

1. Tofigh AM, Kavyani A, Abdollahi SM, Kazemzabeh G, Salman D. Fetus in fetu: Report of a case and literature review. I Journal of Surgery. 2008;6:e94-e96. https://doi.org/10.1016/j.ijsu.2007.04.010

2. Gunaydin M, Celik FC, Tander B, Bozkurter AT. Two cases of fetus in fetu. J Pediatric Surgery. 2011;46:E9E12. https://doi.org/10.1016/j.jpedsurg.2011.05.012

3. YR Yu, J Espinoza, DK Mehta, SG Keswani, TC Lee. Perinatal diagnosis and management of oropharyngeal fetus in fetu: A case report. J Clin Ultrasound. 2018;46(4):286-291. https://doi.org/10.1002/jcu.22528

4. RH Lewis. Foetus in foetus and the retroperitoneal teratoma. Arch Dis Child. 1960;36. https://doi. org/10.1136/adc.36.186.220

5. Willis Rupert A. The structure of a teratoma. J Pathology Bacteriology 1935;40(1). https://doi. org/10.1002/path.1700400102

6. GhazleH,KimberlyD.FetusinFetu.JDMS.2009;25:272276. https://doi.org/10.1177/8756479309344099

7. Miura S, Miura K, Yamamoto T, Yamanaka M, Saito K, Hirabuki T et al. Origin and Mechanisms of Formation of Fetus-in-Fetu: Two Cases With Genotype and Methylation Analyses. Am J Med Genet Part A. 2006;140A:1737-1743. https://doi. org/10.1002/ajmg.a.31362

8. Peterson CM, Buckley C, Holley S, Menias CO. Teratomas: a multimodality review. Curr Probl Diagn Radiol. 2012;41:210-219. https://doi.org/10.1067/j. cpradiol.2012.02.001

9. Escobar MA, Rossman JE, G Michale. Fetus-infetu: report of a case and a review of the literatura. J Pediatric Surgery. 2008;43:943-946. https://doi. org/10.1016/j.jpedsurg.2008.01.061

10. Ianniruberto A, Rossi P, Ianniruberto M, Rella G, Ciminelli V: Sonographic prenatal diagnosis of intracranial fetus in fetu. Ultrasound Obstet Gynecol 2001;18:67-68. https://doi.org/10.1046/j.14690705.2001.00371.x

11. Beaudoin S, Gouizi G, Mezzine S, Wann AR, BarbetP. Mediastinal Fetus in fetu case report and embryological discussion. Fetal Diagn Ther. 2004;19(5):453-455. https://doi.org/10.1159/000078999

12. Parashari UC, Luthra G, Khanduri S, Bhadury S, Upadhyay D. Diagnostic dilemma in a neglected case of fetus-in-fetu solved with Magnetic Resonance Imaging and MDCT - a case. report and review of literatura. J Radiol Case Rep. 2011;5(10): 29-37. https://doi.org/10.3941/jrcr.v5i10.833

13. Majhi AK, Saha K, Karmarkar M, Karmarkar KS. Fetus in fetu - A mystery in medicine. Scient World J. 2007;7:252-257. https://doi.org/10.1100/tsw.2007.56

14. Basu A, Jagdish S, Iyengar KR, Basu D. Fetus-in-fetu or differentiated teratomas. Indian J Pathol Microbiol. 2006;49(4): 563-565.

15. CC Lee, KL Liu, YM Tsang, SJ Chen, HM Liu. Fetus in fetu in an adult: diagnosis by computed tomography imaging. J Formos Med Assoc. 2005;104(3):203205.

16. Kimmel DL, Moyer EK, Peale AR, Winborne LW, Gotwals E. A cerebral tumor containing five human fetuses; a case of fetus in fetu. Anat Rec.1950;106(2):141-165. https://doi.org/10.1002/ ar.1091060205

17. Azam N, Mishra BN, Majhi SK, Joshi RK, Pahi PP. Fetus in fetu presenting as suprarenal mass: a case report. Int Surg J. 2017;4(4):1490-1493. https://doi. org/10.18203/2349-2902.isj20171169

18. Reddy RK, Kannaiyan L, Srirampur S, Malleboyina $\mathrm{R}$, Irfan GM, Sharab H, et al. Thoracic fetus in fetu. J Indian Assoc Pediatr Surg. 2012;17(4):178-179. https://doi.org/10.4103/0971-9261.102344

19. E Borges, JE Lim-Dunham, A Vade. Fetus in fetu appearing as a prenatal neck mass. J Ultrasound Med. 2005;24(9):1313-1316. https://doi.org/10.7863/ jum.2005.24.9.1313

20. Thakral CL, Maji DC, Sajwani MJ. Fetus-in-fetu: a case report and review of the literature. J Pediatr Surg.1998;33(9):1432-1434. https://doi.org/10.1016/ S0022-3468(98)90029-X

21. V Kapoor, L Flom, CR Fitz. Oropharyngeal fetus in fetu. Pediatr Radiol. 2004;34(6):488-491. https://doi. org/10.1007/s00247-003-1103-6

22. JI Yi, Song Bo, Chen Siyuan, Jaing X, Yang G, Gao $\mathrm{X}$, et al. Fetus in Fetu in the Scrotal Sac: Case Report and Literature Review. md-journal. 2015;94(32). https://doi.org/10.1097/MD.0000000000001322

23. Mustafa G, Mirza B, Iqbal S, Sheikh A. A Case of Fetus in Fetu. APSP J Case Rep. 2012;3(2).

24. Mills P1, Bornick PW, Morales WJ, Allen M, GilbertBarness E, Johnson PK, Quintero R; Ultrasound prenatal diagnosis of fetus in fetu; Ultrasound Obstet Gynecol. 2001;18(1):69-71. https://doi.org/10.1046/ j.1469-0705.2001.00469.x

25. Hopkin KL, Dickson PK, Ball TI, Ricketts RR, O'Shea PA, Abramovsky CR. Fetus-in-fetu with malignant recurrence. J Pediatr Surg. 1997;32(10):1476-1479. https://doi.org/10.1016/S0022-3468(97)90567-4

26. Hoeffel CC, Nguyen KQ, Phan HT, Truong NH, Nguyen TS, Tran TT, Fornes P. Fetus in Fetu: A Case Report and Literature Review. Pediatrics. 2000;105(6):1335-1344. https://doi.org/10.1542/peds.105.6.1335

27. Blumberg NA. Abdominal parasitic twin: an unusual cause of duodenal obstruction. Br J Surg. 1980;67(9):675676. https://doi.org/10.1002/bjs.1800670924 